Bericht: Univ.-Prof. Prim. DDr. Peter Fischer, Psychiatrische Abteilung des Donauspitals Wien

Die Originalarbeit „Ärztlicher Personalbedarf in der Ver-

sorgungspsychiatrie - eine "Bottom-Up" Personalbedarfsberechnung am Beispiel der Psychiatrischen Abteilung des Donauspitals in Wien" ist erschienen in der Zeitschrift „Neuropsychiatrie" 1/2016, Seite 10-17, DOI 10.1007/ s40211-016-0170-5, CSpringer Verlag

Stationäre Versorgung

\title{
Ärztlicher Personalbedarf in der Psychiatrie
}

Die Gesellschaft nimmt gegenwärtig in Kauf, dass psychiatrisch schwerkranke Menschen aus Personalmangel nicht State-of-the-Art behandelt werden.

Die Aufgaben einer modernen Psychiatrie sind mit denen der kustodialen Psychiatrie in zentralen Großkrankenanstalten vor 1980 nicht zu vergleichen. Psychotherapie und Soziotherapie sind hoch wirksame Therapien, die jedoch sehr personalintensiv sind. Die durch die Psychiatrie Personalverordnung bereit gestellten Personalressourcen sind heutigen Qualitätsanforderungen nicht mehr gewachsen, sodass der erhebliche Therapiefortschritt der letzten Jahrzehnte nicht bei den schwerstkranken Menschen der Versorgungspsychiatrie ankommt.

Nach den Statistiken des Österreichischen Gesundheitsministeriums arbeiten bezogen auf 100 Betten in psychiatrischen Abteilungen nur etwa $40 \%$ soviel Ärztinnen und Ärzte verglichen mit anderen medizinischen Fachabteilungen. Diese personelle Benachteiligung der Psychiatrie, die übrigens eine Vernachlässigung psychisch Kranker auch nach 1945 bedeutet, wird leider kaum thematisiert. Auch im Strategieplan „Psychische Gesundheit" des Hauptverbandes der Sozialversiche- rungsträger von 2011 finden sich keine Hinweise auf die Notwendigkeit von Verbesserungen der Personalsituation der Psychiatrien.

Wenn an Psychiatrien viel weniger Ärzte und Ärztinnen als an anderen medizinischen Abteilungen arbeiten, ist auch die Wahrscheinlichkeit eines Gespräches mit einem Arzt an psychiatrischen Abteilungen notgedrungen viel geringer als an internen, chirurgischen, HNO-, Haut-, Augen- etc. Abteilungen. Die heutige Versorgungspsychiatrie ist daher gegen den Wunsch der Patienten und der Ärzte und Pflegepersonen eine vorwiegend psychopharmakologische. Wir und unsere Patienten erwarten aber zu Recht und durch alle klinischen Behandlungsleitfäden gedeckt auch Beziehungsarbeit, Gespräche und spezifisches psychotherapeutisches Herangehen.

Die Gesellschaft nimmt schon viel zu lange in Kauf, dass Patientinnen und Patienten der Psychiatrie aus Personalmangel nicht,"State-of-the-Art" behandelt werden. So hätte z. B. jeder Patient oder jede Patientin Anspruch auf eine diagnostische Exploration durch einen Facharzt/einer Fachärztin in den Tagen nach der Aufnahme, um die Diagnose zu hinterfragen oder zu festigen. Auch der Basisbedarf an Beziehungsarbeit und der spezifische psychotherapeutische Bedarf in Gruppentherapien und Einzeltherapien für alle Diagnosegruppen F1 bis F6 des ICD-10 sind gegenwärtig nicht gedeckt.

Es stimmt positiv, dass die Patientenanwaltschaft und die Volksanwaltschaften mit deren Menschenrechtskommissionen sich zunehmend $\mathrm{zu}$ Interessenvertretungen unserer stigmatisierten und benachteiligten $\mathrm{Pa}$ tienten entwickeln und hoffentlich den Druck weiter aufrechterhalten, psychisch kranken Menschen eine faire und menschliche Behandlung nach "State-of-the-Art" zu ermöglichen.

psychopraxis. neuropraxis 2016 · 19:94

DOI 10.1007/s00739-016-0329-7

Online publiziert: 4. Mai 2016

C) Springer-Verlag Wien 2016

Hier steht eine Anzeige. 